\title{
ON THE STRUCTURE OF BALANCED NEAR SEMIRINGS
}

\author{
IVAN CHAJDA AND HELMUT LÄNGER
}

Received 02 January, 2017

\begin{abstract}
The concept of a balanced near semiring was introduced by the authors in [5] in order to describe ring-like structures derived by means of lattices with involution, in particular with complementation. Now we show that every such balanced near semiring has an underlying structure which is a Boolean algebra. Based on this result, we describe ideals of these near semirings. A natural one-to-one correspondence between ideals and congruences is established.
\end{abstract}

2010 Mathematics Subject Classification: 16Y60; 16Y30; 06E25

Keywords: near semiring, antitone involution, balanced near semiring, Boolean algebra, ideal, congruence

As definition of a semiring, we take that of [7] resp. [9]. However, also other non-equivalent definitions can be found in the literature, but the present one is the most appropriate for our reasons because it corresponds in certain sense with various so-called quantum structures, see also [4]. The problem is that in these structures multiplication need not be associative and left distributivity need not be satisfied. This motivated us to introduce the concept of a near semiring, see [4] and [5], which bears some advantages of semirings but the mentioned properties are neglected.

In [5] we investigated mainly near semirings which are, moreover, equipped with an involution and can be derived from lattices having an antitone involution. In some particular cases for lattices with sectional involutions or for ortholattices (see e.g. [1]), we obtained interesting results. Hence, the natural question concerns algebraic constructions of these near semirings, in particular their factorization by congruences. This motivated us to study when a congruence on such an enriched near semiring is determined by its 0-class which is called the congruence kernel. Our first task is to describe the properties of these kernels and to introduce ideals which should correspond to congruences. For this sake, it was useful to reveal the structure of the underlying lattice which turns out to be a Boolean algebra. With this observation in hand, we can use the knowledge on congruences and ideals on Boolean algebras in order to obtain similar results for balanced near semirings.

Support of the research by the Austrian Science Fund (FWF), project I 1923-N25, and the Czech Science Foundation (GAČR), project $15-34697 \mathrm{~L}$, as well as by ÖAD, project $04 / 2017$, is gratefully acknowledged. 
Definition 1. A near semiring is an algebra $(R,+, \cdot, 0,1)$ of type $(2,2,0,0)$ such that $(R,+, 0)$ is a commutative monoid and the following identities are satisfied:

- $(x+y) z \approx x z+y z$,

- $x 0 \approx 0 x \approx 0$,

- $x 1 \approx 1 x \approx x$.

A near semiring $(R,+, \cdot, 0,1)$ is called idempotent if it satisfies the identity $x+x \approx x$.

Let $(R,+, \cdot, 0,1)$ be an idempotent near semiring. Then $(R,+)$ is a semilattice which will be considered as a join-semilattice. The corresponding partial order relation $\leq$ is then defined by $x \leq y$ if $x+y=y(x, y \in R)$. It will be called the induced order of $(R,+, \cdot, 0,1)$. Clearly, 0 is the least element with respect to this order. A unary operation ' on $R$ is called an antitone involution on $(R, \leq)$ if both $x \leq y$ implies $y^{\prime} \leq x^{\prime}$ (for $x, y \in R$ ) and the identity $\left(x^{\prime}\right)^{\prime} \approx x$ is satisfied. Let ${ }^{\prime}$ be an antitone involution on $(R, \leq)$ and put $\mathbf{R}:=\left(R,+, \cdot{ }^{\prime}, 0,1\right)$. If one defines $x \wedge y:=\left(x^{\prime}+y^{\prime}\right)^{\prime}$ for all $x, y \in R$ then $(R,+, \wedge)$ is a lattice with smallest element 0 . The algebra $\mathbf{R}$ is called an ortho near semiring if it satisfies the identity $x+x^{\prime} \approx 0^{\prime}$. Let $\mathbf{R}$ be an ortho near semiring. Then $\left(R,+, \wedge,{ }^{\prime}, 0,0^{\prime}\right)$ is an ortholattice since also the identity $x \wedge x^{\prime} \approx 0$ is satisfied due to the De Morgan laws. We denote this ortholattice by $\mathbb{L}(\mathbf{R})$ and call it the ortholattice corresponding to $\mathbf{R}$. The algebra $\mathbf{R}$ is called a balanced near semiring if it satisfies the identities

$$
0^{\prime} \approx 1 \text { and }\left((x+y)^{\prime}+y\right)^{\prime}+x \approx x .
$$

This is the case if and only if the corresponding ortholattice $\mathbb{L}(\mathbf{R})$ satisfies the identities $0^{\prime} \approx 1$ and

$$
\left((x \vee y) \wedge y^{\prime}\right) \vee x \approx x .
$$

In what follows we are going to specify the ortholattice corresponding to a balanced near semiring $\mathbf{R}$. For this sake we use the method developed by the first author and R. Padmanabhan in [6]. In fact, this result is implicitely contained in [2], but in a rather modified version and with different assumptions. For the reader's convenience we provide the full proof.

Theorem 1. An ortholattice $\mathbf{L}=\left(L, \vee, \wedge,{ }^{\prime}, 0,1\right)$ is a Boolean algebra if and only if it satisfies (0.1).

Proof. In accordance with (0.1) we have

$$
x \wedge\left(x^{\prime} \vee y\right) \approx\left(\left(y \vee x^{\prime}\right) \wedge x\right) \wedge\left(\left(\left(y \vee x^{\prime}\right) \wedge x\right) \vee y\right) \approx\left(\left(y \vee x^{\prime}\right) \wedge x\right) \wedge y \approx x \wedge y,
$$
i.e.

$$
x \wedge\left(x^{\prime} \vee y\right) \approx x \wedge y .
$$

Due to the De Morgan laws also the following holds:

$$
x \vee\left(x^{\prime} \wedge y\right) \approx x \vee y .
$$


Now (0.3) and (0.2) imply

$$
\begin{aligned}
(x \wedge y) \vee z & \approx z \vee(x \wedge y) \approx z \vee\left(z^{\prime} \wedge(x \wedge y)\right) \approx z \vee\left(\left(z^{\prime} \wedge x\right) \wedge y\right) \approx \\
& \approx z \vee\left(\left(z^{\prime} \wedge(z \vee x)\right) \wedge y\right) \approx z \vee\left(z^{\prime} \wedge((x \vee z) \wedge y)\right) \approx z \vee((x \vee z) \wedge y) \approx \\
& \approx((x \vee z) \wedge y) \vee z,
\end{aligned}
$$

i.e.

$$
(x \wedge y) \vee z \approx((x \vee z) \wedge y) \vee z .
$$

Due to the De Morgan laws,

$$
(x \vee y) \wedge z \approx((x \wedge z) \vee y) \wedge z .
$$

Now (0.5) and (0.4) imply

$$
\begin{aligned}
(x \vee y) \wedge z & \approx((x \wedge z) \vee y) \wedge z \approx(x \wedge z) \vee(((x \wedge z) \vee y) \wedge z) \approx \\
& \approx((y \vee(x \wedge z)) \wedge z) \vee(x \wedge z) \approx(y \wedge z) \vee(x \wedge z) \approx(x \wedge z) \vee(y \wedge z) .
\end{aligned}
$$

Hence $\mathbf{L}$ is a distributive ortholattice, i.e. a Boolean algebra. The converse statement is evident.

Corollary 1. An ortho near semiring $\mathbf{R}=\left(R,+, \cdot,{ }^{\prime}, 0,1\right)$ is a balanced near semiring if and only if it satisfies the identity $0^{\prime} \approx 1$ and its corresponding ortholattice $\mathbb{L}(\mathbf{R})$ is a Boolean algebra.

Example 1. Every Boolean algebra $\left(B, \vee, \wedge,{ }^{\prime}, 0,1\right)$ is a balanced near semiring whose multiplication coincides with the operation of lattice meet.

There exist balanced near semirings whose multiplication does not coincide with the meet operation of the corresponding Boolean algebra as can be seen from the following example:

Example 2. If $\mathbf{B}=\left(B, \vee, \wedge,{ }^{\prime}, 0,1\right)$ is a Boolean algebra and

$$
x y:= \begin{cases}x \wedge y & \text { if }\{x, y\} \cap\{0,1\} \neq \varnothing \text { and } \\ y & \text { otherwise }\end{cases}
$$

then $\left(B, \vee, \cdot,{ }^{\prime}, 0,1\right)$ is a balanced near semiring different from $\mathbf{B}$ in case $|B|>2$. This can be seen as follows: Let $a, b, c \in B$. It is easy to check that $(a+b) c=a c+b c$ in case $\{a, b, c\} \cap\{0,1\} \neq \varnothing$. If $\{a, b, c\} \cap\{0,1\}=\varnothing$ then we have $a+b \neq 0$ and hence $(a+b) c=c=c+c=a c+b c$. Moreover, in case $|B|>2$ we have $a a^{\prime}=$ $a^{\prime} \neq 0=a \wedge a^{\prime}$ if $a \neq 0,1$.

Theorem 2. The balanced near semiring $\mathbf{R}=\left(R,+, \cdot{ }^{\prime}, 0,1\right)$ of Example 2 is simple.

Proof. Without loss of generality, assume $|R|>2$. Let $\mathbb{L}(\mathbf{R})=\left(R, \vee, \wedge,{ }^{\prime}, 0,1\right)$, $\Theta \in \operatorname{Con} \mathbf{R}$ and $(a, b) \in \Theta$ with $a \neq b$.

Put $c:=a \wedge b$ and $d:=a \vee b$. Then $(c, d) \in \Theta$ and $c<d$. Put $e:=c \vee d^{\prime}$. Then 
$(e, 1) \in \Theta$. Now $e=1$ would imply $c=(c \vee d) \wedge\left(c \vee d^{\prime}\right)=c \vee d \geq d$, a contradiction. Therefore $e \neq 1$. If $e=0$ then $(x, 1)=(x \vee 0, x \vee 1) \in \Theta$ for all $x \in R$ which implies $\Theta=R^{2}$. Hence assume $e \neq 0$. Then $(x, e)=(x 1, x e) \in \Theta$ for all $x \in R \backslash\{0\}$. Moreover, $e^{\prime} \in R \backslash\{0\}$ and $\left(e^{\prime}, 0\right)=\left(1 \wedge e^{\prime}, e \wedge e^{\prime}\right) \in \Theta$. This shows $\Theta=R^{2}$ also in this case completing the proof of the theorem.

It was shown by the authors in [5] that balanced near semirings $\mathbf{R}$ are congruence regular, i.e. every congruence $\Theta$ on $\mathbf{R}$ is fully determined by every single class $[a] \Theta$, in particular by its 0 -class $[0] \Theta$ which is called the kernel of $\Theta$. Hence, by describing congruence kernels we in fact obtain a complete description of the congruences on R.

Remark 1. A non-empty subset $I$ of the base set $B$ of a Boolean algebra $\mathbf{B}=$ $\left(B, \vee, \wedge,{ }^{\prime}, 0,1\right)$ is called a Boolean ideal of $\mathbf{B}$ if it satisfies the following conditions for all $a, b \in B$ :

- $a \in I$ implies $a \wedge b \in I$,

- $a, b \in I$ implies $a \vee b \in I$.

Let $\operatorname{Id} \mathbf{B}$ denote the set of all Boolean ideals of $\mathbf{B}, \mathbf{I d} \mathbf{B}$ the lattice $(\operatorname{Id} \mathbf{B}, \subseteq)$ of all Boolean ideals of $\mathbf{B}$, Con $\mathbf{B}$ the set of all congruences on $\mathbf{B}$ and $\mathbf{C o n} \mathbf{B}$ the congruence lattice $(\mathrm{Con} \mathbf{B}, \subseteq$ ) of $\mathbf{B}$. It is well-known (see e.g. [8]) that $\Theta \mapsto[0] \Theta$ and $I \mapsto$ $\Theta(I):=\left\{(x, y) \in B^{2} \mid\left(x \wedge y^{\prime}\right) \vee\left(x^{\prime} \wedge y\right) \in I\right\}$ are mutually inverse isomorphisms between Con B and Id B.

Now we define ideals in balanced near semirings.

Definition 2. Let $\mathbf{R}=\left(R,+, \cdot,^{\prime}, 0,1\right)$ be a balanced near semiring and $I$ a nonempty subset of $R$. Then $I$ is called an ideal of $\mathbf{R}$ if it satisfies the following conditions for all $a, b, c \in R$ :

(i) $a \in I$ implies $\left(a^{\prime}+b\right)^{\prime} \in I$,

(ii) $a, b \in I$ implies $a+b \in I$,

(iii) $\left(a^{\prime}+b\right)^{\prime},\left(a+b^{\prime}\right)^{\prime} \in I$ implies $\left((a c)^{\prime}+(b c)\right)^{\prime},\left((c a)^{\prime}+(c b)\right)^{\prime} \in I$.

Let Id $\mathbf{R}$ denote the set of all ideals of $\mathbf{R}, \mathbf{I d} \mathbf{R}$ the ideal lattice $(\operatorname{Id} \mathbf{R}, \subseteq)$ of $\mathbf{R}$, Con $\mathbf{R}$ the set of all congruences on $\mathbf{R}$ and $\mathbf{C o n} \mathbf{R}$ the congruence lattice (Con $\mathbf{R}, \subseteq$ ) of $\mathbf{R}$.

This means that the ideals of a balanced near semiring $\mathbf{R}$ are in fact those Boolean ideals of the corresponding Boolean algebra $\mathbb{L}(\mathbf{R})$ which satisfy condition (iii). Putting $b=0$ in (iii) one obtains $I R \cup R I \subseteq I$.

Remark 2. It can be easily seen that if a balanced near semiring is a Boolean algebra then the concepts of ideals coincide since then multiplication coincides with meet.

Recall that a variety $\mathcal{V}$ is called congruence permutable if $\Theta \circ \Phi=\Phi \circ \Theta$ for all $\mathbf{A} \in \mathcal{V}$ and all $\Theta, \Phi \in \mathbf{C o n} \mathbf{A}$, congruence distributive if $(\Theta \vee \Phi) \wedge \Psi=(\Theta \wedge$ 
$\Psi) \vee(\Phi \wedge \Psi)$ for all $\mathbf{A} \in \mathcal{V}$ and all $\Theta, \Phi, \Psi \in \operatorname{Con} \mathbf{A}$, arithmetical if it is both congruence permutable and congruence distributive and congruence regular if for all $\mathbf{A}=(A, F) \in \mathcal{V}$ and for all $\Theta, \Phi \in \operatorname{Con} \mathbf{A},[a] \Theta=[a] \Phi$ implies $\Theta=\Phi$.

Remark 3. If $\mathbf{R}$ is a balanced near semiring then $\operatorname{Con} \mathbf{R}$ is a sublattice of $\operatorname{Con} \mathbb{L}(\mathbf{R})$ and $\mathbf{I d} \mathbf{R}$ is a sublattice of $\mathbf{I d} \mathbb{L}(\mathbf{R})$. Since the variety of Boolean algebras is arithmetical and congruence regular, the same is true for the variety of balanced near semirings (cf. [5]).

Due to the congruence regularity of $\mathbf{R}$ we have a one-to-one correspondence between ideals of $\mathbf{R}$ and congruences on $\mathbf{R}$.

Theorem 3. Let $\mathbf{R}=\left(R,+, \cdot{ }^{\prime}, 0,1\right)$ be a balanced near semiring. Then $\Theta \mapsto[0] \Theta$ and $I \mapsto \Theta(I):=\left\{(x, y) \in R^{2} \mid\left(x^{\prime}+y\right)^{\prime}+\left(x+y^{\prime}\right)^{\prime} \in I\right\}$ are mutually inverse isomorphisms between $\mathbf{C o n} \mathbf{R}$ and $\mathbf{I d} \mathbf{R}$.

Proof. Let $a, b, c \in R, \Theta \in \operatorname{Con} \mathbf{R}$ and $I \in \operatorname{Id} \mathbf{R}$.

Since $\Theta \in \operatorname{Con} \mathbb{L}(\mathbf{R})$ we have $[0] \Theta \in \operatorname{Id} \mathbb{L}(\mathbf{R})$. Thus $[0] \Theta$ satisfies (i) and (ii). Hence we need only prove (iii). If $\left(a^{\prime}+b\right)^{\prime},\left(a+b^{\prime}\right)^{\prime} \in[0] \Theta$ then $\left(a \wedge b^{\prime}\right) \vee\left(a^{\prime} \wedge b\right) \in[0] \Theta$ and hence $(a, b) \in \Theta([0] \Theta)=\Theta$. This shows $(a c, b c),(c a, c b) \in \Theta=\Theta([0] \Theta)$ and hence $\left((a c)^{\prime}+(b c)\right)^{\prime}=(a c) \wedge(b c)^{\prime} \in[0] \Theta$ and $\left((c a)^{\prime}+(c b)\right)^{\prime}=(c a) \wedge(c b)^{\prime} \in[0] \Theta$ proving (iii). This shows $[0] \Theta \in \operatorname{Id} \mathbf{R}$.

Since $I \in \operatorname{Id} \mathbb{L}(\mathbf{R})$ we have $\Theta(I) \in \operatorname{Con} \mathbb{L}(\mathbf{R})$. We need only prove the compatibility of $\Theta(I)$ with multiplication. If $(a, b) \in \Theta(I)$ then $\left(a^{\prime}+b\right)^{\prime},\left(a+b^{\prime}\right)^{\prime} \in I$ and hence $\left((a c)^{\prime}+(b c)\right)^{\prime},\left((c a)^{\prime}+(c b)\right)^{\prime} \in I$ and, due to symmetry, also $((a c)+$ $\left.(b c)^{\prime}\right)^{\prime},\left((c a)+(c b)^{\prime}\right)^{\prime} \in I$. This shows

$$
\left((a c) \wedge(b c)^{\prime}\right) \vee\left((a c)^{\prime} \wedge(b c)\right),\left((c a) \wedge(c b)^{\prime}\right) \vee\left((c a)^{\prime} \wedge(c b)\right) \in I
$$

and hence $(a c, b c),(c a, c b) \in \Theta(I)$. Therefore $\Theta(I) \in \operatorname{Con} \mathbf{R}$.

The rest follows from Remarks 1 and 3.

Remark 4. Theorem 3 shows that ideals of a balanced near semiring $\mathbf{R}$ coincide with congruence kernels, not only within the underlying Boolean algebra $\mathbb{L}(\mathbf{R})$ but also within $\mathbf{R}$ itself.

In the following theorem we describe principal ideals in balanced near semirings.

If $\mathbf{R}=\left(R,+, \cdot{ }^{\prime}, 0,1\right)$ is a balanced near semiring and $a, b \in R$ then let $\Theta(a, b)$ denote the congruence on $\mathbf{R}$ generated by $(a, b)$ and $I(a)$ denote the principal ideal of $\mathbf{R}$ generated by $a$. Moreover, let $P_{1}(\mathbf{R})$ denote the set of all unary polynomial functions on $\mathbf{R}$.

Theorem 4. Let $\mathbf{R}=\left(R,+, \cdot{ }^{\prime}, 0,1\right)$ be a balanced near semiring and $a \in R$. Then

$$
I(a)=\left\{p(a) \mid p \in P_{1}(\mathbf{R}), p(0)=0\right\} .
$$

Proof. We first prove that $I(a)$ corresponds to $\Theta(a, 0)$. Since $[0] \Theta(a, 0)$ is an ideal of $\mathbf{R}$ containing $a$ we have $I(a) \subseteq[0] \Theta(a, 0)$. Since $\Theta(I(a))$ is a congruence 
on $\mathbf{R}$ containing $(a, 0)$ we have $\Theta(a, 0) \subseteq \Theta(I(a))$. Both assertions together show that $I(a)$ corresponds to $\Theta(a, 0)$. Now let $b \in R$. Then $b \in I(a)$ if and only if $(b, 0) \in \Theta(a, 0)$. Since the variety of balanced near semirings is congruence permutable, $\Theta(a, 0)$ coincides with the least reflexive compatible relation $R(a, 0)$ containing $(a, 0)$ (cf. [10]). According to [3], $(b, 0) \in R(a, 0)$ if and only if there exists a $p \in P_{1}(\mathbf{R})$ with $(p(a), p(0))=(b, 0)$. Hence $b \in I(a)$ if and only if there exists a $p \in P_{1}(\mathbf{R})$ with $p(0)=0$ and $p(a)=b$ completing the proof of the theorem.

It is evident that Id $\mathbf{R}$ is a complete lattice with greatest element $R$ and, according to Theorem 3 with smallest element $\{0\}$. Moreover, meet coincides with settheoretical intersection and for $I, J \in \operatorname{Id} \mathbf{R}, I \vee J$ is the ideal generated by $I \cup J$. Due to Theorem 3, IdR is isomorphic to $\mathbf{C o n} \mathbf{R}$ and hence algebraic.

We recall the concept of a pseudocomplement in a lattice $\mathbf{L}=(L, \vee, \wedge)$ with smallest element 0 . The element $b$ of $L$ is called the pseudocomplement of $a \in L$ if $b$ is the greatest element $x$ of $L$ with $a \wedge x=0$. The lattice $\mathbf{L}$ is called pseudocomplemented if every of its elements has a pseudocomplement.

Now we can prove the following:

Theorem 5. The ideal lattice of a balanced near semiring is pseudocomplemented.

Proof. Let $\mathbf{R}=\left(R,+, \cdot{ }^{\prime}, 0,1\right)$ be a balanced near semiring and $I \in \operatorname{Id} \mathbf{R}$. Since $\mathbf{R}$ is congruence distributive according to Remark 3, Id R is distributive. Since $\mathbf{I d R}$ is also algebraic, meet distributes over arbitrary joins (see e.g. [8]). If $S:=\{J \in \operatorname{Id} \mathbf{R} \mid$ $I \wedge J=\{0\}\}$ and $K:=\bigvee S$ then

$$
I \wedge K=I \wedge \bigvee S=\bigvee\{I \wedge J \mid J \in S\}=\bigvee\{0\}=\{0\}
$$

which shows that $K$ is the greatest element of $(S, \subseteq)$ and hence the pseodocomplement of $I$.

In the following let $I^{*}$ denote the pseudocomplement of the ideal $I$ of $\mathbf{R}$ in Id R.

Recall the concept of factor congruences of an algebra. Let $\mathbf{A}=(A, F)$ be an algebra and $\Theta, \Phi \in$ Con $\mathbf{A}$. Then $(\Theta, \Phi)$ is called a pair of factor congruences of $\mathbf{A}$ if $x \mapsto([x] \Theta,[x] \Phi)$ is an isomorphism from $\mathbf{A}$ to $(\mathbf{A} / \Theta) \times(\mathbf{A} / \Phi)$. It is well-known that this is equivalent to the fact that $\Theta \circ \Phi=\Phi \circ \Theta, \Theta \vee \Phi=\nabla$ and $\Theta \wedge \Phi=\Delta$.

Definition 3. Let $\mathbf{R}$ be a balanced near semiring and $I, J \in \operatorname{Id} \mathbf{R}$. Then we call $(I, J)$ a pair of factor ideals of $\mathbf{R}$ if $(\Theta(I), \Theta(J))$ is a pair of factor congruences of R.

Theorem 6. Let $\mathbf{R}=\left(R,+,,^{\prime}, 0,1\right)$ be a balanced near semiring and $a \in R$. Then $\left(I(a), I\left(a^{\prime}\right)\right)$ is a pair of factor ideals of $\mathbf{R}$ if and only if $I(a) \cap I\left(a^{\prime}\right)=\{0\}$.

Proof. From Theorem 3 we know that $I \mapsto \Theta(I)$ is an isomorphism from Id R to $\mathbf{C o n} \mathbf{R}$ and from Remark 3 we have that any two congruences of $\mathbf{R}$ commute. 
Moreover, $I(a) \vee I\left(a^{\prime}\right)=R$ since $1=a+a^{\prime} \in I(a) \vee I\left(a^{\prime}\right)$. Hence $\left(I(a), I\left(a^{\prime}\right)\right)$ is a pair of factor ideals of $\mathbf{R}$ if and only if $\Theta(I(a)) \cap \Theta\left(I\left(a^{\prime}\right)\right)=\Delta$, i.e. $I(a) \cap I\left(a^{\prime}\right)=$ $\{0\}$.

Corollary 2. Let $\mathbf{R}=\left(R,+, \cdot,^{\prime}, 0,1\right)$ be a balanced near semiring. Then for each $x \in R,\left(I(x), I\left(x^{\prime}\right)\right)$ is a pair of factor ideals of $\mathbf{R}$ if and only if the following condition is satisfied:

If $x \in R, p, q \in P_{1}(\mathbf{R}), p(0)=q(0)=0$ and $p(x)=q\left(x^{\prime}\right)$ then $p(x)=0$.

Proof. Let $a \in R$. According to Theorem 6, $\left(I(a), I\left(a^{\prime}\right)\right)$ is a pair of factor ideals of $\mathbf{R}$ if and only if $I(a) \cap I\left(a^{\prime}\right)=\{0\}$. If $I(a) \cap I\left(a^{\prime}\right)=\{0\}, p, q \in P_{1}(\mathbf{R}), p(0)=$ $q(0)=0$ and $p(a)=q\left(a^{\prime}\right)$ then, according to Theorem 4, $p(a) \in I(a)$ and $q\left(a^{\prime}\right) \in$ $I\left(a^{\prime}\right)$ and hence $p(a)=0$, i.e. (0.6) holds. If, conversely (0.6) holds and $b \in I(a) \cap$ $I\left(a^{\prime}\right)$ then, again according to Theorem 4 , there exist $p, q \in P_{1}(\mathbf{R})$ with $p(a)=b$ and $q\left(a^{\prime}\right)=b$ whence $b=p(a)=0$ according to (0.6) showing $I(a) \cap I\left(a^{\prime}\right)=\{0\}$.

Remark 5. In case $|B|>2$ the balanced near semiring from Example 2 does not satisfy (0.6) since for $a \in B \backslash\{0,1\}$ and $p(x)=q(x):=x a$ for all $x \in B$ we have $p, q \in P_{1}(\mathbf{B}), p(0)=q(0)=0 a=0$ and $p(a)=a a=a=a^{\prime} a=q\left(a^{\prime}\right)$, but $p(a)=$ $a \neq 0$. On the contrary, consider the balanced near semiring from Example 1 which is in fact a Boolean algebra. Since Boolean algebras are in one-to-one correspondence with unitary Boolean rings and every unary polynomial function in a Boolean ring is of the form $p(x)=b x+c$ for some $b, c \in B$, where + denotes the symmetric difference, we can rewrite it in the language of Boolean algebras as $p(x)=(x \wedge d) \vee$ $\left(x^{\prime} \wedge e\right) \vee f$ for some $d, e, f \in B$. Since we ask $p(0)=0$, we obtain $e \vee f=0$ whence $e=f=0$, i.e. $p(x)=x \wedge d$. If now $a \in B, p(x)=x \wedge d$ and $q(x)=x \wedge g$ with some $g \in B$ and $p(a)=q\left(a^{\prime}\right)$ then $p(a)=p(a) \wedge q\left(a^{\prime}\right) \leq a \wedge a^{\prime}=0$ proving that (0.6) holds in this case.

Definition 4. We define the annihilator $\operatorname{Ann}(A)$ of a subset $A$ of a balanced near semiring $\mathbf{R}=\left(R,+, \cdot,^{\prime}, 0,1\right)$ as the greatest ideal $I$ of $\mathbf{R}$ with $I \cap A \subseteq\{0\}$ if this ideal exists. We will write $\operatorname{Ann}(a)$ instead of $\operatorname{Ann}(\{a\})$.

Ann $(A)$ need not exist as the following example shows:

Example 3. In the four-element Boolean algebra considered as a balanced near semiring $\mathbf{R}=\left(\left\{0, a, a^{\prime}, 1\right\},+, \cdot, 0,1\right)$ with join as addition and meet as multiplication $A n n(1)$ does not exist since the ideals $I$ of $\mathbf{R}$ with $I \cap\{1\} \subseteq\{0\}$ are $\{0\},\{0, a\}$ and $\left\{0, a^{\prime}\right\}$ and none of them is the greatest one.

We have $A n n(\varnothing)=A n n(0)=R, A n n(R)=\{0\}$ and if $A \subseteq B \subseteq R$ and $A n n(A)$ and $A n n(B)$ exist then $A n n(B) \subseteq A n n(A)$.

As mentioned above, Id $R$ is infinitely join-distributive. Hence for an ideal $I$ of $\mathbf{R}$ the annihilator $\operatorname{Ann} n(I)$ of $I$ always exists and coincides with $I^{*}$. For annihilators of ideals we have the following easy results: 
Proposition 1. Let $\mathbf{R}=\left(R,+, \cdot,{ }^{\prime}, 0,1\right)$ be a balanced near semiring, $I \in \operatorname{Id} \mathbf{R}$ and $a \in R$. Then the following hold:

(i) $I \subseteq \operatorname{Ann}(\operatorname{Ann}(I))$

(ii) $\operatorname{Ann}(\operatorname{Ann}(\operatorname{Ann}(I)))=\operatorname{Ann}(I)$

(iii) $(I, A n n(I))$ is a pair of factor ideals of $\mathbf{R}$ if and only if $I \vee A n n(I)=R$.

(iv) $\left(I(a), I\left(a^{\prime}\right)\right)$ is a pair of factor ideals of $\mathbf{R}$ if and only if $a^{\prime} \in A n n(I(a))$.

Proof. (i) and (iii) follow from $I \cap \operatorname{Ann}(I)=\{0\}$. (ii) follows from (i) and from the fact that Ann is antitone. (iv) follows from $I(a) \vee I\left(a^{\prime}\right)=R$ and from the fact that the following are equivalent: $I(a) \cap I\left(a^{\prime}\right)=\{0\}, I\left(a^{\prime}\right) \subseteq \operatorname{Ann}(I(a))$, $a^{\prime} \in \operatorname{Ann}(I(a))$.

\section{REFERENCES}

[1] G. Birkhoff, Lattice theory, 3rd ed., ser. American Mathematical Society Colloquium Publications. American Mathematical Society, Providence, R.I., 1979, vol. 25.

[2] I. Chajda, H. Länger, and R. Padmanabhan, "Single identities forcing lattices to be Boolean," Math. Slovaca, (to appear).

[3] I. Chajda, Algebraic theory of tolerance relations. Olomouc: Univerzita Palackého Olomouc, 1991.

[4] I. Chajda and H. Länger, "Commutative basic algebras and coupled near semirings," Soft Comput., vol. 19, no. 5, pp. 1129-1134, 2015.

[5] I. Chajda and H. Länger, "Near semirings and semirings with involution," Miskolc Math. Notes, vol. 17, no. 2, pp. 801-810, 2016.

[6] I. Chajda and R. Padmanabhan, "Lattices with unique complementation," Acta Sci. Math. (Szeged), vol. 83, no. 2, pp. 31-34, 2017.

[7] J. S. Golan, The theory of semirings with applications in mathematics and theoretical computer science, ser. Pitman Monographs and Surveys in Pure and Applied Mathematics. Longman Scientific \& Technical, Harlow; copublished in the United States with John Wiley \& Sons, Inc., New York, 1992, vol. 54.

[8] G. Grätzer, Lattice theory: foundation. Birkhäuser/Springer Basel AG, Basel, 2011. [Online]. Available: http://dx.doi.org/10.1007/978-3-0348-0018-1. doi: 10.1007/978-3-0348-0018-1

[9] W. Kuich and A. Salomaa, Semirings, automata, languages, ser. EATCS Monographs on Theoretical Computer Science. Springer-Verlag, Berlin, 1986, vol. 5. [Online]. Available: http://dx.doi.org/10.1007/978-3-642-69959-7. doi: 10.1007/978-3-642-69959-7

[10] H. Werner, "A Mal'cev condition for admissible relations," Algebra Universalis, vol. 3, p. 263, 1973.

Authors' addresses

Ivan Chajda

Palacký University Olomouc, Faculty of Science, Department of Algebra and Geometry, 17. listopadu 12, 77146 Olomouc, Czech Republic

E-mail address: ivan.chajda@upol.cz 


\section{Helmut Länger}

TU Wien, Faculty of Mathematics and Geoinformation, Institute of Discrete Mathematics and Geometry, Wiedner Hauptstraße 8-10, 1040 Vienna, Austria

Palacký University Olomouc, Faculty of Science, Department of Algebra and Geometry, 17. listopadu 12, 77146 Olomouc, Czech Republic

E-mail address: helmut. laenger@tuwien.ac.at 